\title{
Banned Drugs in India: An Introspective Viewpoint
}

\section{Nilutpal Sharma Bora*}

Assistant Professor, NETES Institute of Pharmaceutical Science, Shantipur, Mirza, Dist-Kamrup, Assam, India

*Corresponding Author: Nilutpal Sharma Bora, Assistant Professor, NETES Institute of Pharmaceutical Science, Shantipur, Mirza, Dist-Kamrup, Assam, India.

Received: November 19, 2019; Published: November 26, 2019

DOI: 10.31080/ASPS.2019.03.0445

Post marketing surveillance of drugs has frequently resulted in the unveiling of many drugs which have serious adverse effects on the human body. When there is such emergence of reports of side effects, there is a brisk requirement of calling off the drugs from the pharmacists' shelves. However, in developing countries like India it is observed that even after the ban of a drug, it is prescribed and administered to the patients in some cases. Several factors contribute to this event; and some of them include absence of law enforcement, lack of physician and pharmacist knowledge, no consumer sensitization regarding the ban. Drugs like Rofecoxib, Phenyl propenamide and Nimesulide which are long banned in the United States (by the USFDA) are still available in India. With a majority of the population in India below poverty line, these banned drugs are found to be cheaper alternatives to safe drugs. A lack of enforcement power of regulatory authorities and a lengthy legal process of banning a drug further worsen the conditions and make India a lucrative market where manufacturers can still produce and market banned drugs. Shortage of manpower in the authoritative sector and the existence of a communication gap between the authorities and the healthcare providers further worsen the situation.

In order to implement the disposal of banned drugs, few crucial steps need to be taken up in India. Superior control over the new drugs should be approved and scrutinized by the Drugs Controller General, India (DCGI). Periodical monitoring of manufacturing facilities, proper disbursal of information regarding banned drugs to healthcare professionals, and thwarting the promotion of banned drugs through averts and labeling; are few of the steps that need to be implemented. Efficient pharmacovigilance centers and drug information centers can also provide valuable information to prescribers, pharmacists and patients. Promotion of generic, safer alternatives of banned drugs can also benefit the pubic due to their safety and cost effectiveness. Lastly more campaigns to sensitize the pubic regarding the adverse effects of banned drugs, along with stringent laws for violators can effectively reduce the use of banned drugs in developing nations like India.

\section{Volume 3 Issue 12 December 2019 (C) All rights are reserved by Nilutpal Sharma Bora.}

